\title{
Deformation Properties of TiNi Shape Memory Alloy
}

\author{
H. Tobushi, P.H. Lin*, K. Tanaka**, C. Lexcellent*** and A. Ikai****
}

Department of Mechanical Engineering, Aichi Institute of Technology, Yagusa-cho, Toyota 470-03, Japan * Department of Mechanical Engineering, Southeast University, Nanjing 210018, China

** Department of Aerospace Engineering, Tokyo Metropolitan Institute of Technology, Hino, Tokyo 191, Japan

*** Laboratoire de Mécanique Appliquée R. Chaléat, associé au CNRS, UFR Sciences et Techniques, La Bouloie, Route de Gray, Besançon cedex 25030, France

**** Graduate School, Aichi Institute of Technology

\begin{abstract}
In order to describe the deformation properties due to the martensitic transformation and the R-phase transformation of TiNi shape memory alloy, a thermomechanical constitutive equation considering the volume fractions of induced phases associated with both transformations is developed. The proposed constitutive equation expresses well the properties of the shape memory effect, pseudoelasticity and recovery stress.
\end{abstract}

\section{INTRODUCTION}

In shape memory alloy (SMA), the residual strain of several percent disappears by heating which is called the shape memory effect (SME) and the transformed strain recovers by unloading which is called the pseudoelasticity (PE) or superelasticity. These deformation properties occur due to the martensitic transformation (MT). In TiNi SMA, the properties occur not only due to the MT but also due to the rhombohedral phase transformation (RPT)[1-3]. Therefore, the deformation behavior of TiNi SMA due to the MT and the RPT is very complex.

In order to design SMA elements, the stress-strain-temperature relationship is necessary. For this purpose, the authors proposed the theory which was useful for the practical application and described well the deformation behavior due to the MT[4-7]. In the theory, a stress-strain-temperature equation was used considering a volume fraction of induced phase. Furthermore they proposed a constitutive equation which described the deformation properties due to the RPT[8-10].

In the present study, based on the previous studies, a constitutive equation which describes the deformation behavior associated with the MT and the RPT is proposed. Based on the experimental data, the material parameters are determined and the calculated results are compared with the experimental results. It is ascertained that the proposed theory is useful for design of SMA elements.

\section{THEORY}

\subsection{Constitutive Relationship}

In the present study, the stress-strain-temperature relationship is discussed in the case of uniaxial tension. For a constitutive equation which describes the deformation behavior associated with the MT and the RPT, the constitutive equation which is developed based on the relationship in the previous studies[4-9] is proposed.

We assume the following constitutive equation which describes the thermomechanical behavior of the material.

$$
\dot{\sigma}=D \dot{\varepsilon}+\Theta \dot{T}+\Omega \dot{\xi}+\Psi \dot{\eta}
$$


We assume the following transformation kinetics which governs the progress of the transformation.

$$
\begin{aligned}
& \xi=\Xi(\sigma, T) \\
& \eta=H(\sigma, T)
\end{aligned}
$$

In these equations, $\sigma, \varepsilon$ and $T$ represent the stress, strain and temperature, respectively. The internal state variables $\xi(0 \leq \xi \leq 1)$ and $\eta(0 \leq \eta \leq 1)$ represent the volume of the martensitic phase and the rhombohedral phase (R-phase), respectively. The volume fractions $\xi$ and $\eta$ satisfy the conditions: $0 \leq \xi+\eta \leq 1$. The volume fractions $\xi$ and $\eta$ are respectively prescribed by the transformation kinetics (2) and (3).

The dot on the letter in Eq.(1) denotes the time derivate. The coefficients $D$ and $\Theta$ represent the modulus of elasticity and the thermoelastic constant, respectively. The quantity $|\Omega / D|$ or $|\Psi / D|$ represent the transformation strain range due to the MT or the RPT, which denotes the maximum recoverable strain and can be evaluated from the crystallographic difference between the parent (austenite) phase and the martensitic phase or between the parent phase and the R-phase, respectively[4-9].

\subsection{Transformation Kinetics}

We assume the concrete form of the transformation kinetics (2) and (3) which describes the progress of the transformation. Firstly, with respect to the MT, we employ the following form, the same as the previous paper[4-7].

$$
\begin{aligned}
& \dot{\xi} /(1-\xi)=b_{M} c_{M} \dot{T}-b_{M} \dot{\sigma} \geq 0 \\
& -\dot{\xi} / \xi=b_{A} c_{A} \dot{T}-b_{A} \dot{\sigma} \geq 0
\end{aligned}
$$

Equation (4) is applied to the MT and Eq.(5) to its reverse transformation. The coefficients $b_{M}, C_{M}, b_{A}$ and $c_{A}$ are the material constants. If we assume that these material parameters are constant and integrate Eqs.(4) and (5), we obtain the following equations.

$$
\begin{aligned}
& \xi=1-\exp \left\{b_{M} \mathcal{C}_{M}\left(M_{s}-T\right)+b_{M} \sigma\right\} \\
& \xi=\exp \left\{b_{A} C_{A}\left(A_{s}-T\right)+b_{A} \sigma\right\}
\end{aligned}
$$

$M s$ and $A s$ respectively represent the temperatures at which the MT and its reverse transformation start under stress-free conditions. The MT starts or completes when $\xi=0$ or $\xi=1$ in Eq.(2), respectively. These conditions are represented by the curves on the stress-temperattire plane and are referred to as the transformation starting line and the transformation completing line. With respect to Eqs.(6) and (7), the equations for these lines are obtained by taking $\xi=0$ or $\xi=0.99$, instead of 1 , as usual in metallurgy. Each transformation progresses in the respective region between these two lines, which is called the transformation zone.

Secondly, with respect to the RPT, we employ the following form, the same as the previous paper $[8,9]$

$$
\begin{aligned}
& \eta=b_{M}{ }^{\prime} C_{M}{ }^{\prime}\left(T-M s^{\prime}\right)-b_{M^{\prime}} \sigma \\
& \eta=1+b_{A^{\prime}} C_{A^{\prime}}\left(A s^{\prime}-T\right)+b_{A^{\prime}} \sigma
\end{aligned}
$$

Equation (8) is applied to the RPT and Eq.(9) to its reverse transformation. The coefficients $b_{M^{\prime}}{ }^{\prime}, C_{M^{\prime}}{ }^{\prime}, b_{A^{\prime}}{ }^{\prime}$ and $C_{A}{ }^{\prime}$ are the material constants. $M s^{\prime}$ and $A s^{\prime}$ respectively represent the temperatures at which the RPT and its reverse transformation start under stress-free conditions. The RPT starts or completes when $\eta=0$ or $\eta=1$ in Eq.(3), respectively. These conditions are represented by the curves on the stresstemperature plane.

At the low temperatures of $T \leq M s^{\prime}$ and $T \leq A s^{\prime}$, the starting and completing stresses of the RPT and its reverse transformation are almost constant. Thus we assume that the respective starting and completing lines of the transformation are expressed as

$$
\begin{aligned}
& \sigma=\sigma_{N o^{\prime}} \\
& \sigma=\sigma_{M o^{\prime}}-1 / b_{M^{\prime}}
\end{aligned}
$$

for the RPT and

$$
\begin{aligned}
& \sigma=\sigma_{A 0^{\prime}} \\
& \sigma=\sigma_{A 0^{\prime}}-1 / b_{A^{\prime}}{ }^{\prime}
\end{aligned}
$$

for its reverse transformation. Therefore the starting and completing lines of the RPT are represented by the respective bilinear lines. Each transformation progresses in the respective region between the two bilinear lines.

\subsection{Stress-Strain Curves}

The stress-strain curves for uniaxial tension at constant temperature are shown in Fig.1. When we 
draw up Fig.1, we consider the following points. If the specimen of TiNi SMA is loaded in tension at constant temperature, the RPT appears at first and the MT follows. During the progress of each transformation, the interface between the induced phase and the parent phase moves like Luder's bands in the yield stage of mild steel[11].

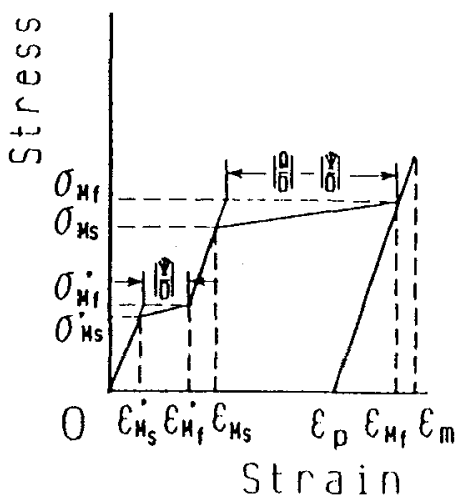

(a) $T<A s, \quad \varepsilon_{n}>\varepsilon_{i f}$

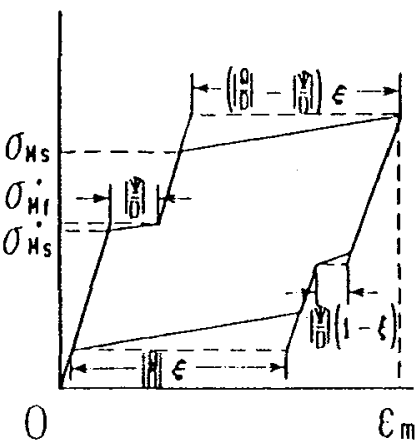

Strain

(b) $T>A_{f}, \sin <\varepsilon_{i f}$

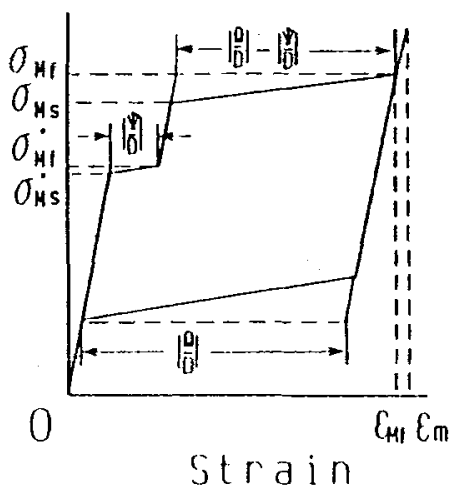

(c) $T>A f$, Eif

Fig. 1 Stress-strain curves at constant temperature $T$ and transformation strain ranges

As shown in Fig. 1 , in the case that the RPT starts at first under the stress $\sigma_{t s} s^{\prime}$, if the volume fraction of the R-phase is $\eta$, the volume fraction of the parent phase is $1-\eta$. If the RPT completes under the stress $\sigma_{M f^{\prime}}, \eta=1$. In the case that stress increases after completion of the RPT and the MT starts under the stress $\sigma_{\pi / s}$, if the volume fraction of the martensitic phase is $\xi$, the volume fraction of the R-phase $\eta$ is $1-\xi$. If the MT completes under the stress $\sigma_{\text {kff }}, \xi=1$ and $\eta=0$.

\section{RESULTS AND DISCUSSION}

\subsection{Coefficients and Transformation Lines}

We carried out the tensile tests at a constant temperature and the heating tests under a constant strain for Ti-55.3wt\%Ni SMA wire, $0.75 \mathrm{~mm}$ in diameter. The respective values of coefficients for the MT and the RPT determined based on the experimental results are shown in Tables 1 and 2. Because the thermoelastic effect is small in the range treated in the present paper, we assume $\Theta=0$. Although the modulus of elasticity $D$ depends on $T, \xi$ and $\eta$, we assume that $D$ is constant because we are describing the overall behavior of SMA using a simple model and are proposing the model useful for design of SMA elements. The transformation strain range $|\Omega / D|$ due to the MT increases in proportion to $T$ and the range $|\Psi / D|$ due to the RPT decreases. Therefore if $D$ is assumed constant, the coefficients $\Omega$ and $\Psi$ are expressed as follows.

Table 1 Coefficients for the MT

\begin{tabular}{|c|c|c|c|c|c|c|c|c|}
\hline $\mathrm{D}$ & $\mathrm{I}$ & $\mathrm{n}$ & $\mathrm{CH}_{\mathrm{H}}$ & $\mathrm{C}_{A}$ & $\mathrm{~b}_{\mathrm{M}}$ & $\mathrm{b}_{A}$ & $\mathrm{M}_{\mathbf{S}}$ & $A_{\mathbf{S}}$ \\
\hline $\mathrm{GPa}$ & $\mathrm{MPa} / \mathrm{K}$ & $\mathrm{MPa}$ & $\mathrm{MPa} / \mathrm{K}$ & $\mathrm{MPa} / \mathrm{K}$ & $1 / \mathrm{MPa}$ & $1 / \mathrm{MPa}$ & $\mathrm{K}$ & $\mathrm{K}$ \\
\hline 60 & -6 & -1962 & 6.125 & 8.8 & -0.132 & 0.132 & 272.5 & 318 \\
\hline
\end{tabular}

Table 2 Coefficients for the RPT

\begin{tabular}{|c|c|c|c|c|c|c|c|c|c|c|}
\hline D) & $m^{\prime}$ & $n^{\prime}$ & $\mathrm{CM}^{\circ}$ & $\mathrm{c}_{\wedge}$ & $\sigma_{M \dot{0}}$ & $\mathrm{~b}_{M^{\prime}}$ & $1 / \mathrm{MPa})$ & $\mathrm{b}_{\mathrm{A}^{\prime}}$ & $M_{\mathbf{s}}$ & $A s^{\circ}$ \\
\hline$G \mathrm{G} a$ & $\mathrm{MPa} / \mathrm{K}$ & $\mathrm{MPa}$ & $\mathrm{MPa} / \mathrm{K}$ & $\mathrm{MPa} / \mathrm{K}$ & Mia & $T<H_{\mathbf{s}}$ & $T \geqq M_{\mathbf{s}^{\prime}}$ & I/MPa & $k$ & $k$ \\
\hline 60 & 6.19 & -2386 & 15.2 & 17.1 & 25 & -0.091 & -0.028 & 0.025 & 317 & 318 \\
\hline
\end{tabular}




$$
\begin{aligned}
& \Omega=m T+n \\
& \Psi=m^{\prime} T+n^{\prime}
\end{aligned}
$$

The starting lines and the completing lines for the MT and its reverse transformation $M_{s}, M_{f}, A s$ and $A f$ and the starting lines and the completing lines for the RPT and its reverse transformation $M_{s}{ }^{\prime}, M_{f}^{\prime}$, $A s^{\prime}$ and $A f^{\prime}$ determined by using these coefficients are represented by the straight lines on a stresstemperature plane. The reverse transformation lines will be shown in Figs. 3 and 4.

\subsection{Stress-Strain Relationship}

The stress-strain curves with various maximum strain $\mathrm{em}$ for the tensile tests at $\mathrm{T}=303 \mathrm{~K}$ and $333 \mathrm{~K}$ are shown in Fig.2. In Fig.2, thin curves represent the experimental results and thick curves the calculated results. In the case of $T=303 \mathrm{~K}$, residual strain appears after unloading and diminishes by heating under no-stress, showing SME. In the case of $\mathrm{T}=333 \mathrm{~K}$, the transformed strain recovers by unloading, showing PE.

Comparing the thin curves with the thick curves, we find that the calculated results quantitatively describe the overall stress-strain relationship due to the RPT and the MT.
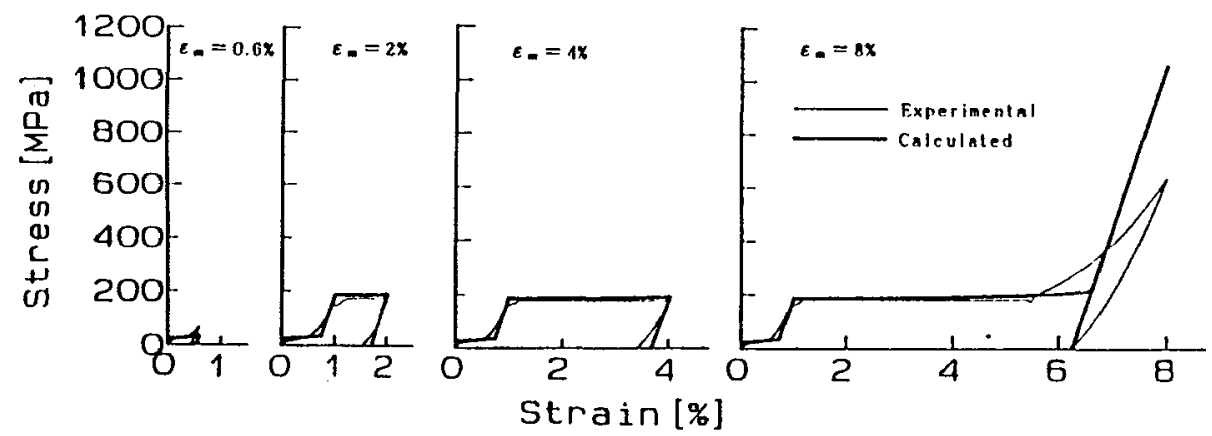

(a) $\mathrm{T}=303 \mathrm{~K}$
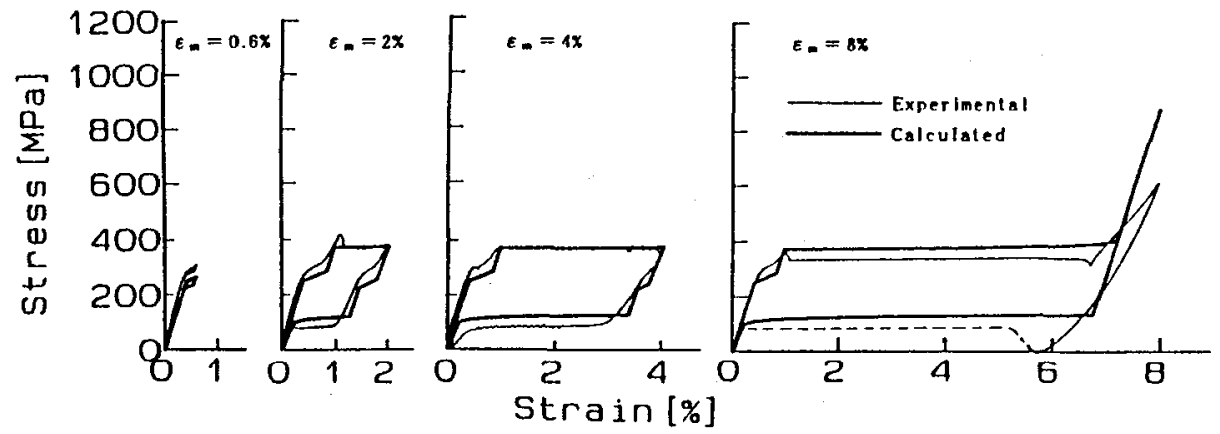

(b) $\mathrm{T}=333 \mathrm{~K}$

Fig.2 Stress-strain curves for the tensile tests at constant temperature

\subsection{Stress-Strain Curves and Recovery Stress}

Stress-strain curves and stress-temperature curves for the heating tests under constant residual strain which appears during loading and unloading processes at $T=303 \mathrm{~K}$ and these under constant maximum strain are shown in Figs. 3 and 4, respectively. In Figs. 3 and 4, thin curves represent the experimental results and thick curves the calculated results. In the stress-temperature diagram in Figs. 3 and 4 , the reverse transformation lines $A s$ and $A_{f}$ for the MT and these $A s^{\prime}$ and $A_{f}{ }^{\prime}$ for the RPT are plotted by chain lines and dashed lines, respectively. As seen from the stress-temperature curves during the heating process in Figs. 3 and 4 , in the case of $\varepsilon_{m} \leq 1 \%$, recovery stress increases along the reverse transformation line for the RPT. If $\varepsilon_{m}$ is in the MT region, recovery stress increases at first along the reverse transformation line for the RPT and increases after that along the reverse transformation line for 
the MT. In this case, if $\varepsilon m$ is large, recovery stress due to the RPT is small and that due to MT is large.

Comparing the thin curves with the thick curves in Figs. 3 and 4, we find that the calculated results quantitatively describe the overall behavior of recovery stress.

\section{CONCLUSIONS}

The thermomechanical constitutive equation considering the volume fractions of induced phase due to the MT and the RPT of TiNi SMA is developed. The proposed constitutive equation expresses well the properties of SME, PE and recovery stress. The proposed theory is useful for design of SMA elements.

\section{REFERENCES}

[1] Miyazaki, S and Otsuka, $K$, Phil Mag. A. 50-3 (1984), 393.

[2] Miyazaki,S and Otsuka,K, Metall Trans, A, 17A (1986), 53.

[3] Miyazaki,S, Kimura,S and Otsuka, K, Phil Mag, A, 57-3 (1988), 467.

[4] Tanaka,K, Res Mech, 18 (1986), 251.

[5] Tanaka,K, Kobayashi,S and Sato, Y, Int J Plasticity, 2 (1986), 59.

[6] Tanaka, $\mathrm{K}$, Tobushi, $\mathrm{H}$ and Iwanaga, $H$, Proc, 31st Japan Cong Mater Research, (1988) 51.

[7] Tanaka, K, Hayashi, T, Itoh, $Y$ and Tobushi,H, Mech Mater, 13 (1992), 207.

[8] Tobushi,H, Tanaka,K, Kimura,K, Hori, T and Sawada, T, JSME inter J,I,35-3(1992) 278.

[9] Sawada, T, Tobushi,H, Kimura,K, Hattori, T, Tanaka, K and Lin Pinghua, JSME Inter J, A, 36-4 (1993), 395.

[10] Lexcellent, $\mathrm{C}$, Tobushi, $\mathrm{H}$, Ziolkowski,A and Tanaka, $\mathrm{K}$, Int $\mathrm{J}$ Pres Ves \& Piping, 58(1994), 51.

[11] Funakubo,H. ed., Shape Memory alloys (Gordon \& Breach Sci, 1987)pp. 39-41.
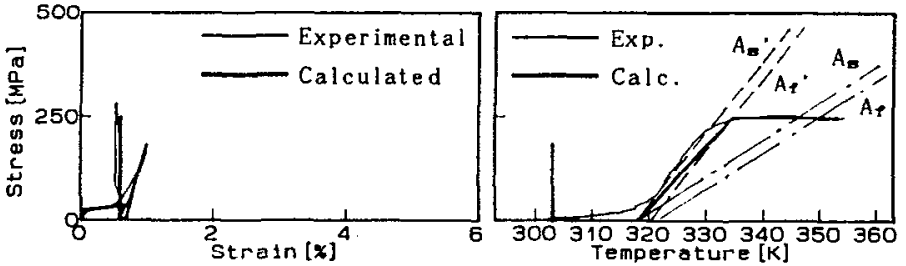

(a) $\varepsilon_{m}=1 \%$

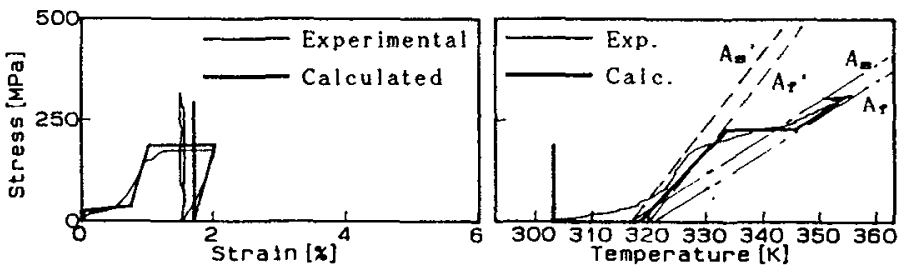

(b) $\varepsilon_{m}=2 \%$

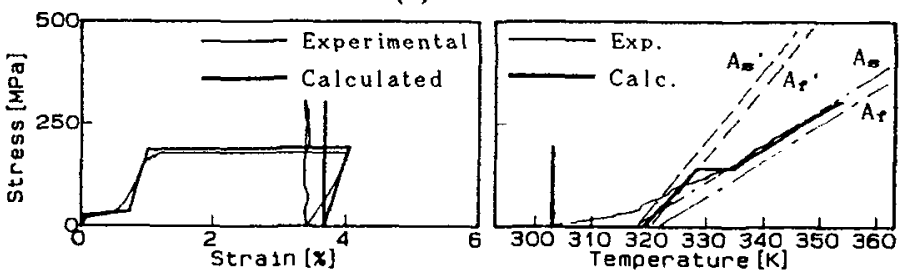

(c) $\varepsilon_{n}=4 \%$

Fig. 3 Relationships of stress versus strain and stress versus temperature under constant residual strain
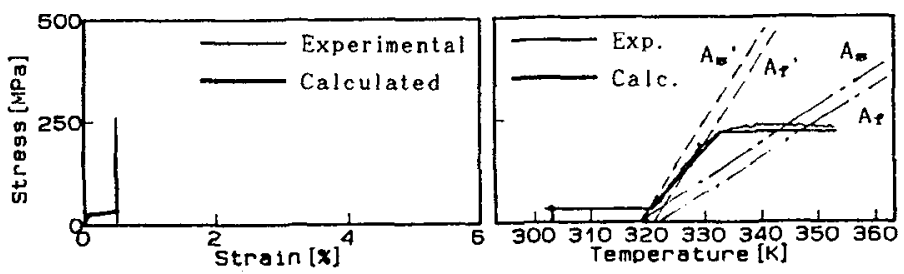

(a) $\epsilon_{m}=0.5 \%$

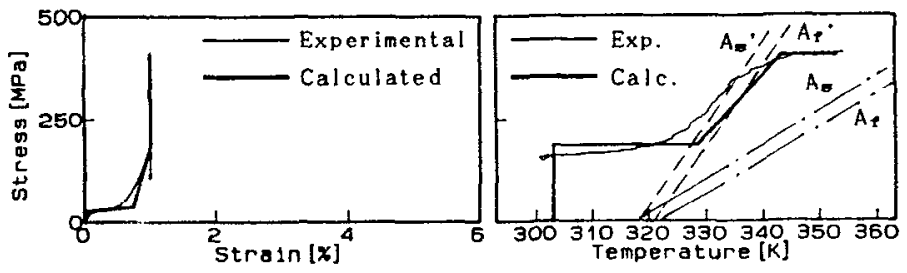

(b) $\varepsilon_{m}=1 \%$

Fig.4 Relationships of stress versus strain and stress versus temperature under constant maximum strain 